\title{
Neural correlates of free recall of "famous events" in a "hypermnestic" individual as compared to an age- and education-matched reference group
}

\author{
Thorsten Fehr ${ }^{1,2,3^{*}}$ (D) Angelica Staniloiu ${ }^{4,6}$, Hans J. Markowitsch ${ }^{4,6}$, Peter Erhard ${ }^{1,3,5}$ and Manfred Herrmann ${ }^{1,2,3}$
}

\begin{abstract}
Background: Memory performance of an individual (within the age range: 50-55 years old) showing superior memory abilities (protagonist PR) was compared to an age- and education-matched reference group in a historical facts ("famous events") retrieval task.

Results: Contrasting task versus baseline performance both PR and the reference group showed fMRI activation patterns in parietal and occipital brain regions. The reference group additionally demonstrated activation patterns in cingulate gyrus, whereas PR showed additional widespread activation patterns comprising frontal and cerebellar brain regions. The direct comparison between PR and the reference group revealed larger fMRI contrasts for PR in right frontal, superior temporal and cerebellar brain regions.

Conclusions: It was concluded that PR generally recruits brain regions as normal memory performers do, but in a more elaborate way, and furthermore, that he applied a memory-strategy that potentially includes executively driven multi-modal transcoding of information and recruitment of implicit memory resources.
\end{abstract}

Keywords: Memory, fMRI, Superior memory, Memory strategy, Experts, Complex cognition

\section{Background}

Aside from the time-based distinction into short-term/ working and long-term memory [1], memory is nowadays partitioned into content-based systems [2, 3]. Long term memory (LTM) has been discussed to be distinguished into episodic-autobiographical memory (memory for personal events or experiences), semantic memory (conscious knowledge of facts, including factual self-knowledge), perceptual memory (conscious familiarity judgments), procedural memory (mechanical, motor-related skills) and priming (higher likelihood of re-identifying previously perceived stimuli) [4-6]. Superior LTM performance has been described to tap into different memory systems. There are reports of individuals

\footnotetext{
*Correspondence: fehr@uni-bremen.de

${ }^{2}$ University of Bremen, Hochschulring 18, 28359 Bremen, Germany

Full list of author information is available at the end of the article
}

with highly superior autobiographical memory $[7,8]$, of semantic memory experts [9-11], and descriptions of specific types of hypermnesia $[12,13]$.

In general, LTM performance might be facilitated by the application of optimal learning strategies $[14,15]$ and/or the existence of elaborated, domain-specific expert knowledge networks [16], to which new information can be both efficiently associated to and recalled from. Parker et al. [7] proposed a specific form of superior memory performance, the hyperthymestic syndrome (HS), which is characterized by superior memory that is assumed to be automatically organized, and not based on explicit mnemonic strategies. It addresses idiosyncratic memory domains; these particular individuals do not necessarily score higher than average on standard memory tests tapping on information that is irrelevant for them. In a similar way, Norman Brown [17] put forth a model of "historical memory" elaboration that assumes 
an association of historical facts of public interest to individually relevant episodic information, which might be related to both the template theory proposed by Gobet and Clarkson [16] and, in case of superior performance, to the HS advanced by Parker et al. [7].

The neural mechanisms for memory encoding and retrieval are still under debate, and especially for superior memory performance, they are still largely unknown. In particular, since the prominent patient H.M. showed selective impairment in consciously encoding and consolidating new facts and events long-term [18-20], these processes were assumed to be mediated by the hippocampus and its adjacent medial temporal brain regions [21-23]. However, other regions of the limbic system situated in medial diencephalon and basal forebrain, equally contribute to these processes [5]. There are also theories suggesting that LTM networks can be associated with highly integrated networks distributed all over the neural system [24-26] and that the hippocampal formation is involved in both conscious and unconscious information processing $[27,28]$.

Functional neuroimaging and clinical studies involving patients with different forms of amnesia as a result of regional brain damage, however, support the idea that different memory systems might recruit at least partially "distinct" brain networks [5, 20, 29]. Aside from limbic structures involved in processes of binding and associating information to LTM, further cortical structures-sometimes referred to as expanded or greater limbic system [30, 31]-such as the retrosplenial cortex and the precuneus were related to processes of imagination, of the representation of memories, and to familiarity [32-34]. These structures might play an important role in both normal, but in particular, in superior LTM performance.

In the present study, we carried out a comprehensive neuropsychological assessment and additionally conducted a functional magnetic resonance imaging (fMRI) study that focussed on a memory retrieval task in an individual (PR) with superior historical facts knowledge ("famous events memory"). The present experimental design was specifically designed to examine the neural correlates of recalling memories related to historical facts. FMRI-contrast included the conditions 'recall of declarative memory content WITH reference to historical facts' and 'recall of declarative memory content WITHOUT reference to historical facts'. It was assumed that PR should show less pronounced activation patterns in frontal (i.e., executive memory organization), but enhanced neural involvement of limbic brain regions (automatic and/or pre-attentive memory organisation) and precuneus (perceptual, imaginative, confidence judgments and/or familiarity based memory strategies) in comparison to an age- and education-matched reference group.

\section{Results \\ Behavioural data}

Before applying parametric t-statistics, a Shapiro-Wilktest (S-W-T) was performed in order to test, whether variables of interest were normal-distributed. In the reference group, the percentage of freely recalled correct answers (consistently shown for both scanner session and post hoc debriefing) was significantly higher in the BASE (test on normal distribution: $\mathrm{S}-\mathrm{W}-\mathrm{T}, \mathrm{W}=.93, p=.45$ ) compared to the TASK (test on normal distribution: S-W-T, W $=.88, p=.13$ )-condition (TASK: $41.7 \pm 8.3 \%$; BASE: $89.6 \pm 7.2 \% ; t$ Test: $\mathrm{t}=15.2, p<.001$; see Fig. $1 \mathrm{a})$, and reference group members performed better than chance level in both TASK- and BASE-conditions (TASK vs. $25 \%$ : $\mathrm{t}=-1.9, p<.05$; BASE vs. $25 \%: \mathrm{t}=-8.6, p<.001$ $[35,36])$. Compared to the reference group, PR showed a higher correct percentage rate in the TASK-condition $(\mathrm{t}=4.1, p<.01$ [35, 36]; see Fig. 1a) and comparable performance in the BASE-condition. All participants showed high performance in the BASE task. As, however, performance-values were normal-distributed (see above) and PR's performance ranged in the middle of the reference-group performances, we rather tend to disclose a ceiling effect. Participants in the reference group showed longer response times for correctly answered TASK-trials (test on normal distribution: $\mathrm{S}-\mathrm{W}-\mathrm{T}, \mathrm{W}=.87, p=.09$ ) compared to BASE-trials (test on normal distribution: $\mathrm{S}-\mathrm{W}-\mathrm{T}, \mathrm{W}=.90, p=.24$ ) during free recall (TASK: $3728 \pm 733$ ms; BASE: $3056 \pm 429 \mathrm{~ms}$; $t=4.4, p<.01$; see Fig. 1b). According to Crawford and Howell [35], PR (TASK: $3599 \mathrm{~ms}$; BASE: $3664 \mathrm{~ms}$ ) did not differ in any response time value from the reference group.

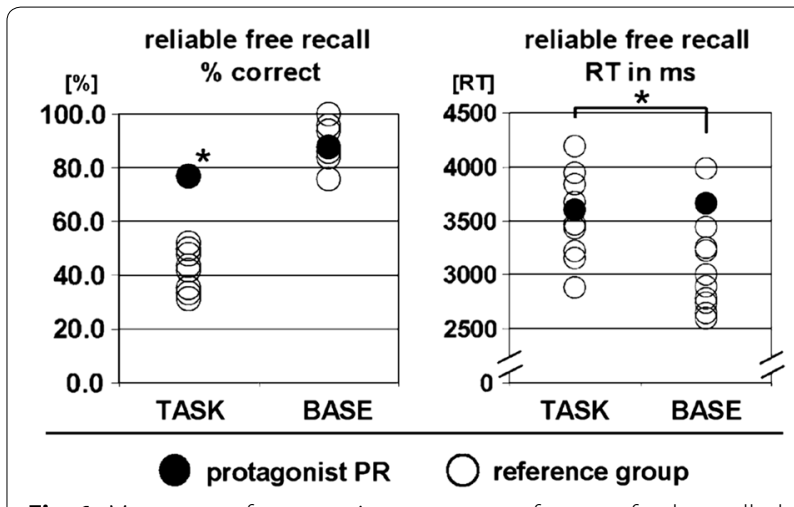

Fig. 1 Memory performance in percentage of correct freely recalled answers (left panel) and the respective mean response times (right panel); asterisks indicate significant differences ( $p<.05$; details, see text) and whiskers indicate standard deviations 
FMRI-data: TASK versus BASE-condition in PR and in reference participants

For the reference group, second level fMRI-analysis for TASK in contrast to BASE-condition revealed activation clusters in the right anterior and left posterior cingulate, bilateral precuneus, cuneus, and lingual gyrus (Fig. 2a; Table 1, column A). Protagonist PR showed activation clusters in widespread bilateral superior, medial, and middle frontal gyri, right postcentral gyrus, left precuneus, right middle and left inferior occipital gyri, left fusiform gyrus, and left cerebellar regions for the contrast TASK versus BASE-condition (Fig. 2b; Table 1, column B).

All PSC-value-distributions were tested for deviations from normal distribution via Shapiro-Wilk-Test: rPCG:
$\mathrm{W}=.92, p=.33$; rSFG: $\mathrm{W}=.86, p=.05$; lMFG: $\mathrm{W}=.98$, $p=.96$; first cluster rMFG: $\mathrm{W}=.94, p=.48$; second cluster rMFG: $.93, p=.42$; rSTG: $\mathrm{W}=.94, p=.47$; rCUL: $\mathrm{W}=.92, p=.29$. The direct comparison between PR and reference group yielded larger contrasts between TASKand BASE-conditions distributed over precentral gyrus, right superior and bilateral middle frontal gyri, right superior temporal gyrus, and the left culmen (Fig. 2c; Table 1, column C). Reference group versus PR showed larger contrasts in left paracentral lobule, right precuneus, and left cuneus (Fig. 2d; Table 1, column D).

In Fig. 3, distributions of difference-values between PSC-values for TASK and BASE-conditions for several ROIs were illustrated for the reference group and

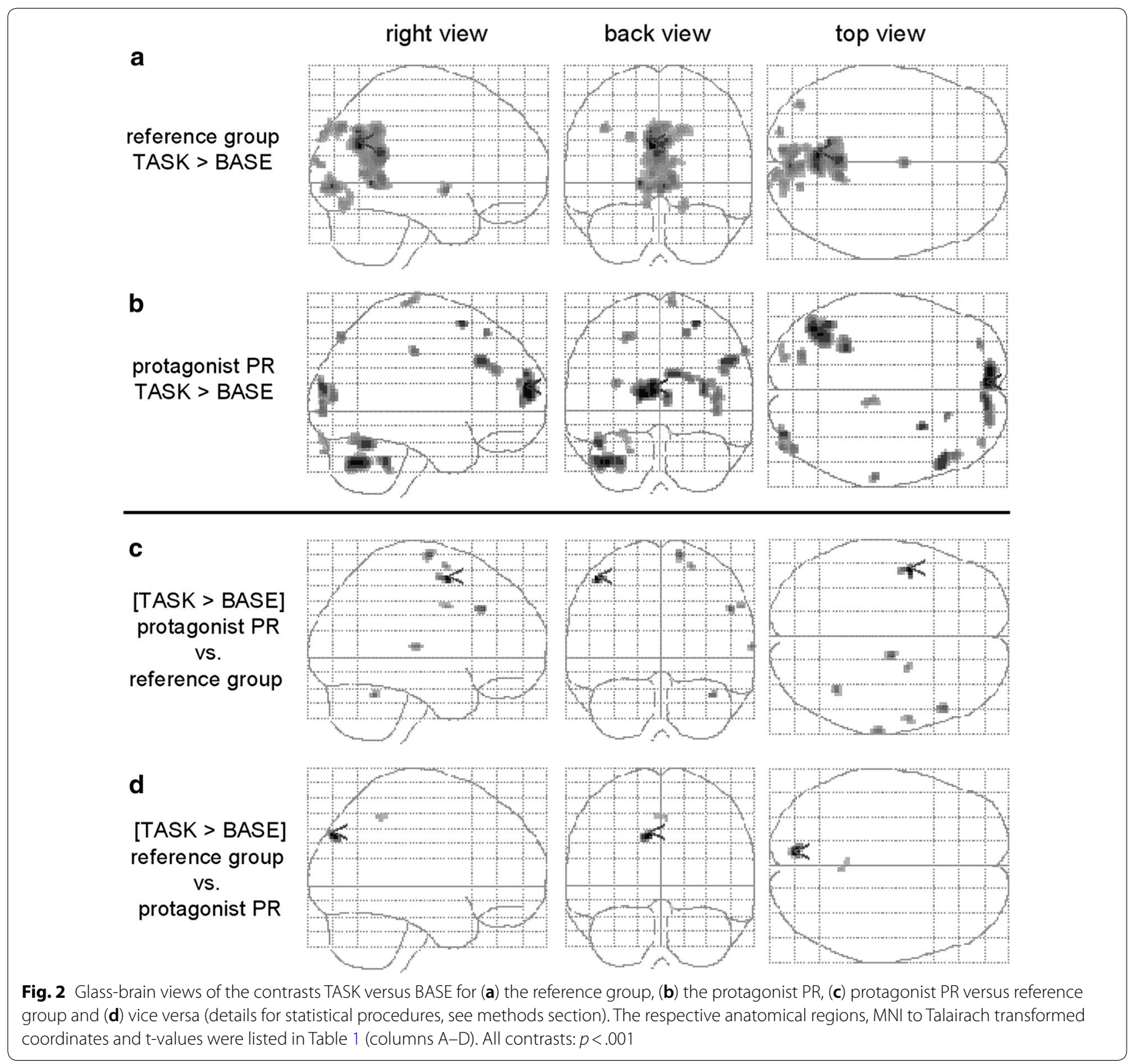




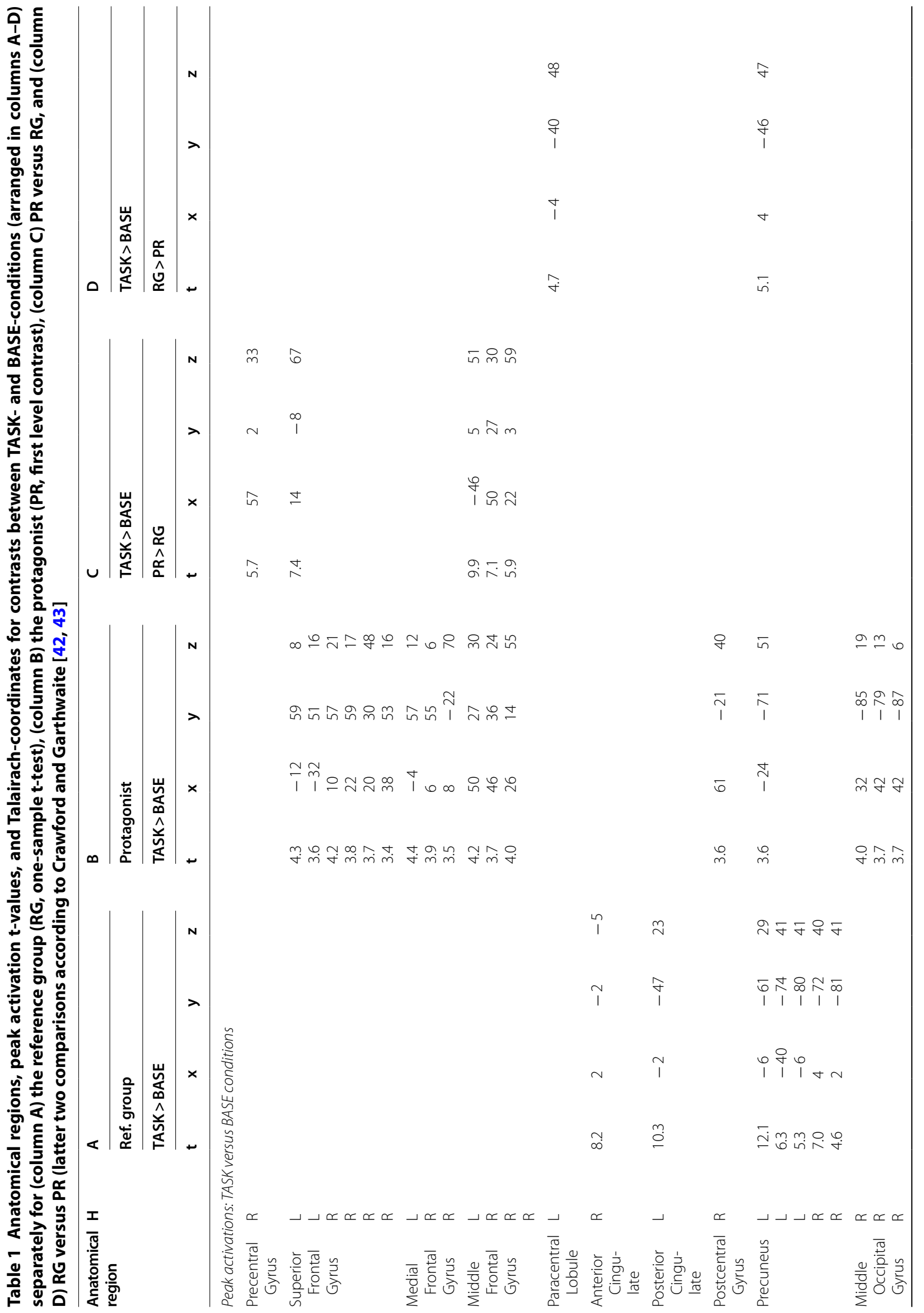




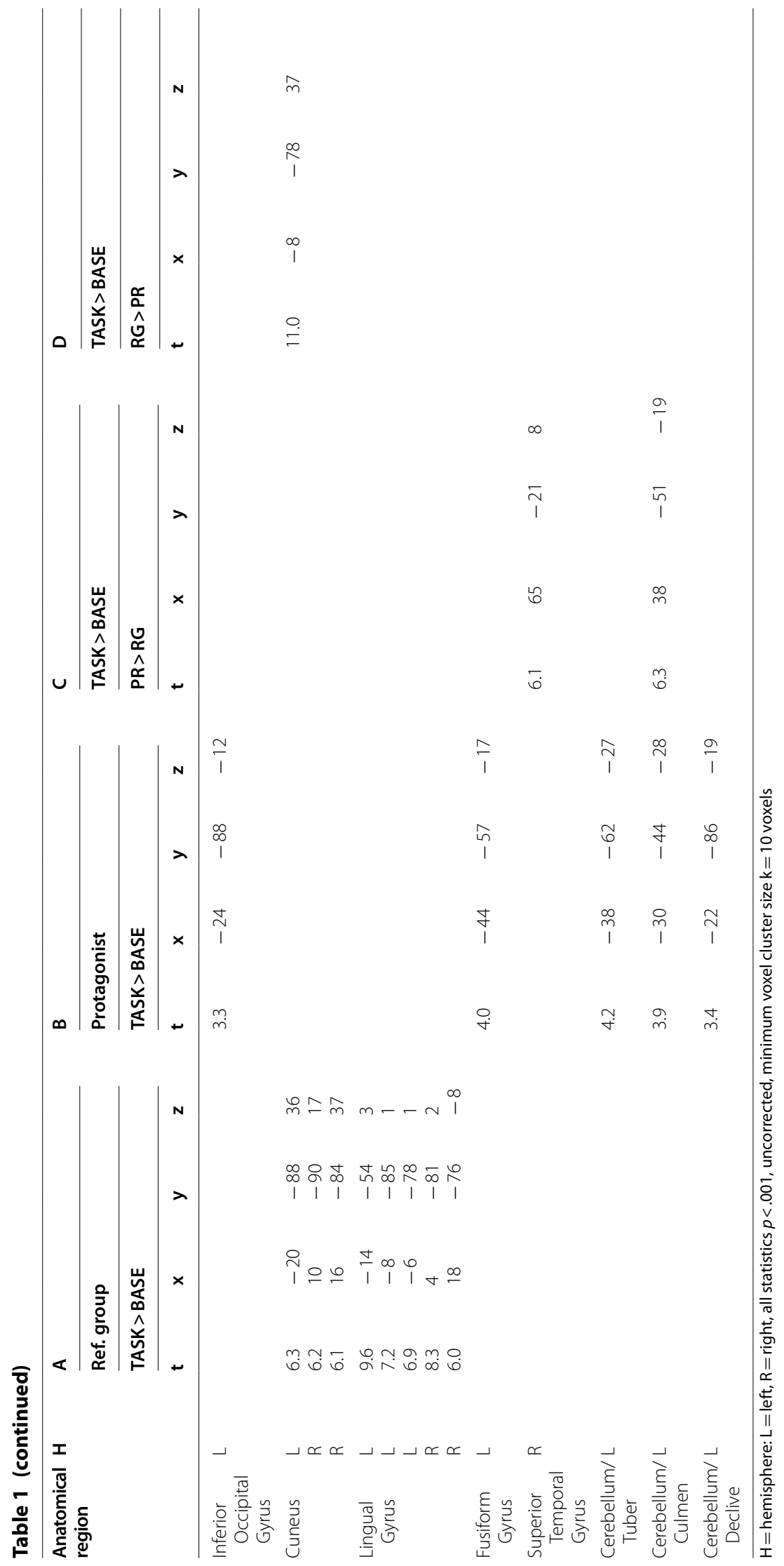




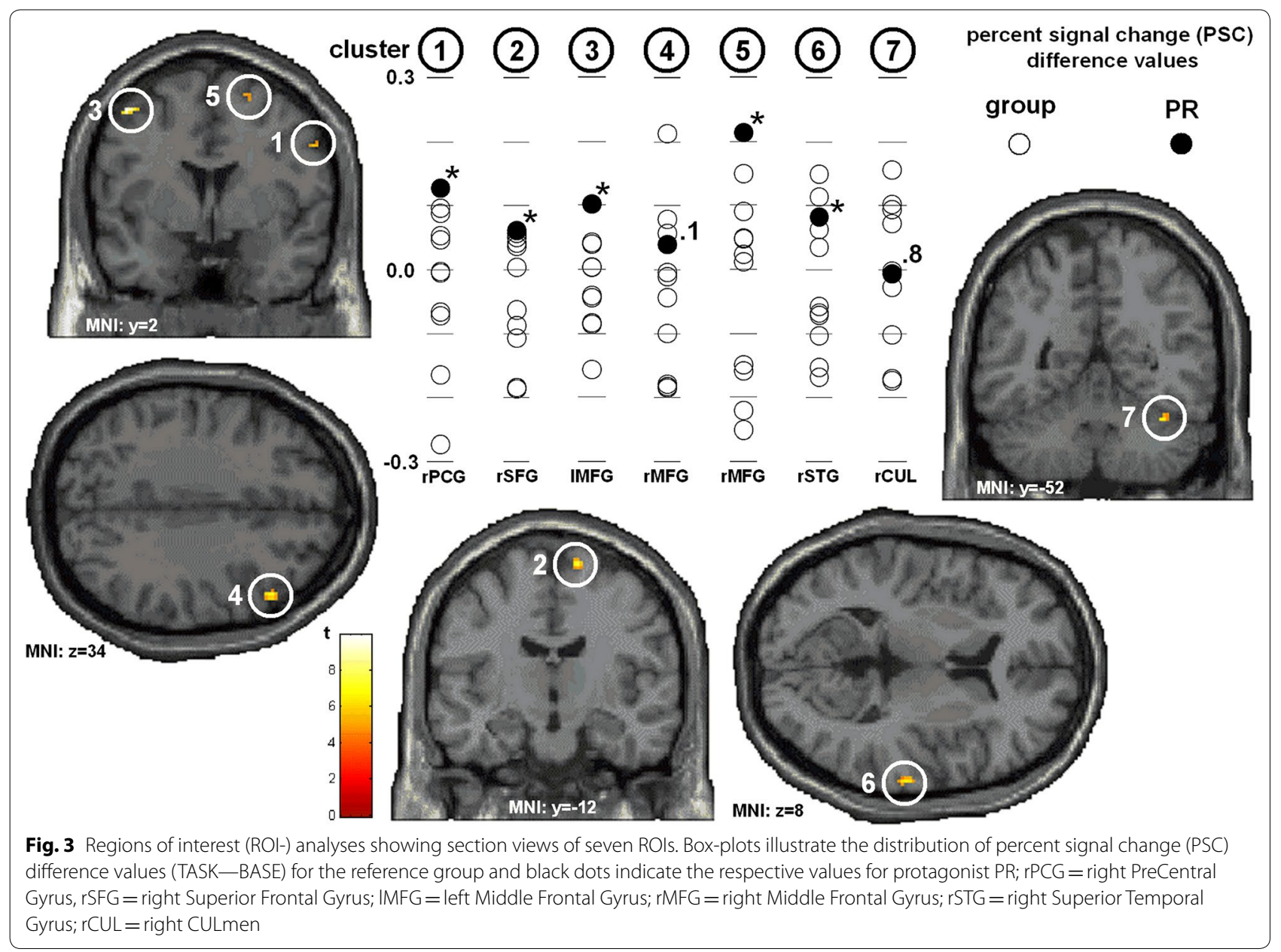

separately for PR. T-Test for single means yielded to individual versus group differences in right precentral gyrus, right superior frontal gyrus, left and right middle frontal gyri, and right superior temporal gyrus.

\section{Discussion}

In the present study, functional neuroimaging was used to compare a protagonist's (PR) superior memory performance in a famous events free retrieval task (contrasted with a semantic non-historical facts free retrieval task serving as baseline) with the performance of a reference group. PR performed significantly better than the reference group and showed activation patterns predominantly distributed over frontal and cerebellar, but also in parietal, occipital and occipito-temporal brain regions. The reference group demonstrated activation patterns in the cingulate cortex, parietal, and occipital brain regions. The direct comparison between PR and the reference group confirmed larger contrasts for frontal and cerebellar regions in PR and for parietal and occipital brain areas in the reference group. It appears that PR predominantly recruited right hemispheric frontal resources potentially related to his superior memory retrieval performance $[44,45]$.

\section{Which type of expert is PR?}

First, PR showed superior memory performance for famous events retrieval, and he scored predominantly at average or above average in most retrograde and anterograde memory tests in the neuropsychological assessment. Only for complex anterograde non-verbal memory processing he scored below average. Incidentally, the case described by Parker et al. [7] also showed impairments in anterograde non-verbal memory tasks. Thus, while PR shares certain similitudes with HS (hyperthymestic syndrome [7]), he presents with unique features, which deviate from the HS prototype. He showed, beside superior historical facts memory, deviations from standards in non-idiosyncratically eminent memory domains. The mnemonic performance of PR can be at least partly understood within the model of Markowitsch and Tulving. Incidentally, Tulving [46] indirectly anticipated later 
models of mnemonic processing, which describe porous boundaries between memory systems [47]. In 1995, Tulving [46] proposed his SPI-model which states that encoding of information follows a regular sequence-that means it is serial in that way that first simple, implicitly functioning, memory systems are engaged and only at the end of the series explicit, episodic encoding occurs. Information then is stored in parallel memory systems in the brain and it can be retrieved independently from the systems used for the encoding process (SPI = Serial, Parallel, Independent). As PR describes his knowledge as "popping out automatically", it can be speculated that according to Tulving's [46] SPI-model, PR retrieves his knowledge "automatically", and therefore more independently from his initial encoding of it, than it likely is the case in most human beings.

In addition, PR showed sub-average performance on tests for executive functions. While this type of performance may be interpreted as a hint towards a savant-syndrome [48], there was no clear clinical, neuropsychological or anamnestic evidence in his case that he fell into this category. He showed a superior intelligence level comparable to the reference group as confirmed by a verbal intelligence test. Furthermore, he scored above average on standardized laboratory tests for assessing social cognition and emotional processing, although he reported some interpersonal difficulties in real-life, which were being addressed in psychotherapy. Incidentally, the case described by Parker et al. [7] also presented with some difficulties in the executive functioning domain. Furthermore, LePort et al. [8] found in a case series of patients with highly superior autobiographical memory abilities a psychological profile indicative of obsessive compulsive tendencies. These tendencies typically are accompanied by a reduction in cognitive flexibility, which gets translated in impaired performance on corresponding neuropsychological tasks. The deficient performance of PR on tasks tapping on cognitive flexibility and the anamnestic reports about PR may speak in favour of obsessive compulsive personality phenotypic traits. These traits may promote an automatic engagement in repetition of mnemonic material of special interest, with consequences for processes of encoding and consolidation. Conclusively, PR cannot be seen comparable to individuals with HS or to savants in the classical sense. It rather appears that he is an expert sharing some features of HS and savant people, but does not fully overlap with any of these prototypes, displaying unique features. In the following, characteristics in fMRI activation patterns are discussed to conclude about the individual mnemonic strategy that PR might have applied to score higher in free historical facts retrieval than the reference group.

\section{Can the present functional neuroimaging data explain PR's profile of superior memory performance?}

The hypothesis that PR should show rather posterior and/ or subcortical instead of frontal activation patterns when compared to the reference group, had to be rejected. The opposite was the case: PR showed predominantly right frontal patterns of larger contrasts between free historical and non-historical semantic facts retrieval. At first glance, this finding appears quite disillusioning in view of the hypotheses postulated in the introduction section, however, combined with the data that PR also showed remarkable cerebellar recruitment, a hybrid mental strategy of memory processing including both explicit and implicit components might be conceivable $[10,48]$. And, this argument also goes with the hybrid classification (between savant and expert) concluded from the neuropsychological testing data as mentioned in the previous section.

\section{Level of processing and multi-modal integration of memory}

There were also exclusive activation patterns in PR not present in the reference group and vice versa, which could in part only be interpreted in an individual (PR) or group-internal (reference-group) way as the direct comparison between PR and the reference group did not reach statistical significance in the respective brain regions. In posterior brain regions, associated with perceptual information processing, PR showed activation patterns rather adjacent to primary (i.e., middle and inferior occipital gyrus, and postcentral gyrus) and less in hetero-modal, medial (i.e., cuneus and lingual gyrus) cortical brain regions as the reference group did. Rather medial activation patterns in the reference group might be related to a higher level complex or abstract memory processing [25]. This finding might also explain why PR scored above average in simple, but below average in complex recognition tasks that usually require a deeper associative memory strategy.

Transcoding and integration of information across processing modalities (i.e., implicit and explicit processing, and verbal, visual, and spatial modalities, etc.), as can be seen for example in synesthesia [49-51] was discussed as a potential feature of both superior expert and savant memory performance involving occipitotemporal regions such as the fusiform gyrus $[9,10]$. The present data showed exclusive fusiform gyrus involvement in PR, but not in the reference group. It should however be mentioned that the direct comparison between groups via between-group t-test did not reveal differences in this region. Nevertheless, in combination with an exclusive cerebellar recruitment in PR, this data might point to an implicit-explicit (and vice 
versa) memory transcoding strategy facilitating superior memory performance in historical facts retrieval, but also in other simple memory processing domains as shown by the neuropsychological assessment.

The stronger involvement of superior temporal gyrus (STG) in PR additionally supports the idea of pronounced multi-modal integration of information in LTM potentially facilitating his retrieval performance. The multi-associative nature of STG has been documented by studies discussing the role of the STG in retrieving both autobiographical events [52-54] and semantic facts, such as public events or "long-established knowledge about the world" [52, 55, 56].

Furthermore, the right superior temporal cortex was thought to be engaged in spatial awareness and exploration [57]. Along this line of argumentation, the stronger recruitment of STG in PR may signify a strategy to navigate through personal past by making use of more elaborated spatial exploration strategies [53]. Recently, Manning and colleagues argued that public semantic memory is supported by both the semantic and episodic memory system [58]. The stronger involvement of the STG in PR in contrast to the reference group might again point to a particular engagement of the episodic memory system in public semantic knowledge processing in the case of PR and other individuals with highly superior autobiographical talents [8,59-61].

\section{Limitations of the present study}

In future studies the number of tasks in the different task-condition should be perfectly matched.

For the present reference group there were 15-25 correct response trials in the TASK-condition left to be modeled for the respective fMRI-statistics. Despite normal distribution and despite numerous studies also reporting reliable data based on a quite low number of trials and/or individuals, this point should be carefully considered for an appropriate interpretation of the here presented data. In future studies additional measurement sessions should be taken into account to extend the number of valid trials. In the present study, we were however forced to balance the available time (PR was only available for a short time period) and test-statistical requirements.

\section{Conclusions}

Specific complex mental processes cannot be inferred directly from functional brain imaging data [62], however, there is evidence that regional brain activation can help to understand the underlying mental principles involved in a certain complex mental process such as the applied visual, verbal or spatial modality, or perceptual and/or executive processing types, and others more [63-65]. It appears that the utilization of individual mental strategies also plays an important role in effective memory processing [61, 66-69]. And, these individual memory strategies can be modulated by executive mental processing as potentially reflected in the pronounced recruitment of frontal brain regions in PR. Furthermore, the present data support the idea that superior mental processing in experts can be facilitated by the conceptually driven recruitment of implicit/procedural memory resources (i.e., potentially reflected by the involvement of cerebellar brain areas [10]). A more detailed assessment of mental strategies in individuals with superior mental performance can provide insights into effective implicit memory usage potentially driven by explicit executive mental processing. Functional neuroimaging can help to evaluate the recruitment of implicit mental resources that are difficult to be assessed by explicit surveys.

\section{Methods \\ Study participants}

The individual protagonist PR (within the age range: 50-55 years old) was a healthy, right handed expert with superior memory abilities.

Applying a test-battery several months before the fMRI-measurement session provided a detailed neuropsychological performance-profile of PR (see Table 2 for details).

The reference group consisted of 10 male adults between 47 and 62 years $(54.6 \pm 4.3$ years.; not differing in age from PR: $\mathrm{t}=-.8, p=.445$; $[35,36]$. All participants were right handed according to a modified version of the Edinburgh Handedness Questionnaire [37], and did not report psychiatric or neurological illness or psychotropic drug treatment. All participants were native German speakers holding a university degree. The participants were familiarized with the assessment environment and their participation was solely motivated by their interest in scientific investigations.

After the fMRI-session, verbal intelligence was examined in all participants with the MWT-B (MehrfachWahl-Wortschatz-Test [38]), for which PR reached 130, and members of the reference group reached $126.0 \pm 13.6$ (test on normal distribution with Shapiro-Wilk-test: $\mathrm{W}=.89, p=.18)$. Here, $\mathrm{PR}$ did not differ from the group $(\mathrm{t}=-.28, p=.79[35,36])$.

The study protocol was designed and performed according to the Helsinki Declaration (1964) and was approved by the Ethics Committee of Bremen University. All participants were informed about the procedure, and gave written consent to participate in the experiment.

\section{Task and stimuli}

The tasks were presented visually. The experimental setup includes two task conditions, a baseline (BASE) and 
Table 2 Protagonist PR was examined with a battery of different test inventories

\begin{tabular}{|c|c|c|}
\hline Mental domains and tests & Score & Interpretation \\
\hline \multicolumn{3}{|l|}{ Attention, concentration } \\
\hline Trail making test $A+B$ & A: 42 s, 1 error; B: 174 s, 0 errors & Below average \\
\hline $\mathrm{d} 2-\mathrm{R}$ test & 122 correct, 12 errors & Average \\
\hline WMS-R, attention and concentration index & 96 & Average \\
\hline \multicolumn{3}{|l|}{ Intelligence } \\
\hline Mehrfach-Wahl-Wortschatz-test B & 34 of 37 (IQ 130) & Above average \\
\hline Wechsler intelligence test raw scores & $24,23,15,34(I Q>125)$ & \\
\hline \multicolumn{3}{|l|}{ Visuo-constructive abilities } \\
\hline Rey-Osterrieth figure (ROF), copy & 36 & Normal \\
\hline \multicolumn{3}{|l|}{ Interference } \\
\hline Color-word-interference test (CWIT) & $12,22,37 \mathrm{~s}$ & Above average \\
\hline \multicolumn{3}{|l|}{ Anterograde memory } \\
\hline ROF, by heart after $1 / 2 \mathrm{~h}$ & 21 & Average \\
\hline WMS-R, general memory & 94 & Average \\
\hline Verbal learning memory test (VLMT) & $\begin{array}{l}63 \text { learning, } 7 \text { interference; } 15+15 \\
\text { in Trials } 6+7,50 / 0 \text { in recognition }\end{array}$ & Above average \\
\hline Doors test & $\begin{array}{l}\text { simple recognition: } A=12 \\
\text { complex recognition: } B=5\end{array}$ & $\begin{array}{l}\text { Above average } \\
\text { Below average }\end{array}$ \\
\hline \multicolumn{3}{|l|}{ Retrograde memory } \\
\hline Semantic: Famous Faces Test (38 pictures) & 30 directly identified, 2 with cues & Above average \\
\hline $\begin{array}{l}\text { Episodic-autobiographical old memory } \\
\text { (EAMT) }\end{array}$ & Gives per epoch well-described examples & Very good \\
\hline $\begin{array}{l}\text { Semantic old famous events } \\
\text { (1970s-1990s) }\end{array}$ & 22 named, 1 recognized, 2 unknown & Very good \\
\hline \multicolumn{3}{|l|}{ Emotion } \\
\hline Mind in the Eyes Test & 19/24 correct & Above average \\
\hline Florida Affect Battery & $\begin{array}{l}\text { Facial Identity Discrimination: } 20 \text { of } 20 \\
\text { Facial Affect Discrimination: } 20 \text { of } 20\end{array}$ & $\begin{array}{l}\text { Above average } \\
\text { Above average }\end{array}$ \\
\hline \multicolumn{3}{|c|}{ Problem solving ability, cognitive flexibility, executive functions, risk taking behavior } \\
\hline Cronin-Golomb concept formation task & $15-16$ of 17 & Good average \\
\hline Category test & $4,5,5$ categories & Below average \\
\hline Tower of Hanoi (4 discs) & 49 moves, $5 \mathrm{~min}, 21 \mathrm{~s}$ & Below average \\
\hline Word fluency (COWAT Test) & $17+10+14$ & Average \\
\hline Wisconsin card sorting test (WCST) & $\begin{array}{l}20 \text { correct, } 12 \text { errors } \\
16 \text { perseveration errors }\end{array}$ & Below average \\
\hline Game of dice test (with 12 moves) & $+800 €$ at finish & Thoughtful strategy \\
\hline \multicolumn{3}{|l|}{ Tendencies for malingering } \\
\hline Rey 15-Item Test & All correct & Inconspicuous \\
\hline Test of memory malingering (TOMM) & Fist trial: 48 of 50 & Inconspicuous \\
\hline Test battery for forensic neuropsychology (TBFN) & 13 correct, 2 false & Inconspicuous \\
\hline Amsterdam short term memory test & Two errors in the first 15 trials & Inconspicuous \\
\hline
\end{tabular}

See details and references to the test battery listed in the Additional file 1: Supplementary online document S1

a task of interest (TASK). Both task conditions included questions in order to test semantic memory performance. BASE-condition tested semantic memory about common knowledge such as for example "How is the head of the Catholic church called?", and the TASK-condition tested semantic memory about public historical facts from contemporary history, for example "Which city hosted the Olympic Summer Games in 1996?”.
The respective questions were presented via a digital projector on a mirror in the scanner tube in the center of the display as a centered text-block (see Fig. 4 for illustration). There was no time limit to think about the correct answer. Responses consisted of pressing the answer button with the right index finger. Individuals, however, were encouraged not to ruminate too long about the answer and they were asked to press a button 


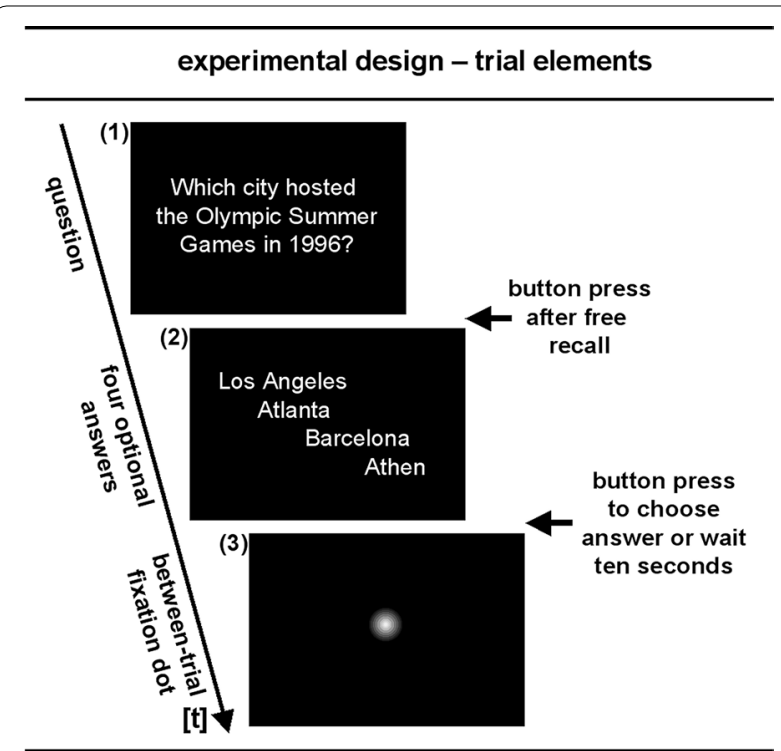

Fig. 4 Experimental trial and trial elements: (1) Question, waiting for button press after free recall or conformation of omission, (2) choosing the correct answer after recall or recognition, or wait ten seconds for the between-trial period, and (3) between-trial fixation-period ( $3500 \pm 500 \mathrm{~ms}$, pseudo-randomised jittered)

with the right index finger, if they either believed to know the correct answer or when they were sure not to know the answer. This procedure was applied to ensure that participants produced free recall and not recognition performance. After pressing the button, four alternative answers (one correct solution) were presented slightly shifted one below the other in the center of the display (see Fig. 4 for illustration). If participants freely recalled the correct answer, they were asked to choose it from the four alternatives. If however, they did not recall the correct answer, but recognized it among the four alternatives, they were allowed to choose the correct one. If they neither recalled nor recognized the correct answer, participants were asked to wait (ten seconds) for the next trial. Between trials a fixation dot was presented for a pseudo-randomly jittered interval ranging between 3000 and $4000 \mathrm{~ms}$.

After the MRI-scanner session, all questions were again presented by a paper-pencil-test. The participants were asked again to answer correctly to all BASE- and TASKquestions and to further provide detailed information about whether they freely recalled, recognized or did not know the correct answers during the fMRI-measurement. BASE- and TASK-trials were only included in behavioural and fMRI-data-analyses, if they were consistently answered correctly during both the fMRI-measurement and the paper-pencil-test, and furthermore, if they were labeled to be freely recalled and not just recognized.
All other trials were neglected in behavioural data analyses and modeled as dummy-trials in the fMRI-analyses (see below).

50 BASE- and 48 TASK-trials were presented in a pseudo-randomized non-stationary probabilistic weighted sequence [39] during one experimental run of $17 \pm 2 \mathrm{~min}$.

\section{FMRI data acquisition and analyses FMRI-data acquisition}

Functional magnetic resonance imaging (fMRI) data were collected on a SIEMENS MAGNETOM scanner (Skyra syngo MR D13, 3 Tesla). The images were acquired using a BOLD weighted gradient echo echoplanar imaging (EPI) sequence (TE $30 \mathrm{~ms}$ ). Forty four slices were acquired in interleaved order with no gap in axial orientation parallel to AC-PC with a GRAPPA accelleration factor of two leading to a TR of $2500 \mathrm{~ms}$. The image volume covered the entire cerebrum and cerebellum. The in plane resolution was $3 \times 3 \mathrm{~mm}^{2}$, the corresponding matrix $64 \times 64,411 \pm 44$ volumes were obtained during the functional run. Structural whole head T1 weighted images were acquired (TR/TE/TI/flip angle $=2400 \mathrm{~m}$ $\mathrm{s} / 2.43 \mathrm{~ms} / 900 \mathrm{~ms} / 8^{\circ}$; matrix $256 \times 256$; slice thickness $1.0 \mathrm{~mm}$; FOV $256 \mathrm{~mm}$; 176 slices) for all participants.

\section{FMRI-data analysis}

Image analysis was performed using additional algorithms for the comparison of single individuals with group-related data (see below), which were implemented in SPM (http://www.fil.ion.ucl.ac.uk/spm/). For each session and participant, images were realigned to the first image in the time series to correct for head motion. The realigned images were spatially normalized into a standard stereotaxic space (Montreal Neurological Institute template) using a 12 parameter affine model. Dimensions after normalising procedures were $79(\mathrm{x}) \times 95(\mathrm{y}) \times 69(\mathrm{z})$ and resulting voxel size was $2 \mathrm{~mm}^{3}$. These spatially normalized images were smoothed to minimize noise and residual differences in gyral anatomy with a Gaussian filter set at $8.0 \mathrm{~mm}$. Prior to the statistical analysis, a temporal high pass filter (250 s) was applied and global effects were removed. Pre-processed data sets were analysed using secondlevel random effects models [40] on the individual parameter estimates.

FMRI data were modelled for different trial element phases (see Fig. 4 for illustration): (1) from the start of task presentation until the first button press (separately for correct, incorrect, and omitted trials), (2) from the display of the four response alternatives to the second button press or until the trial time runs out (separately for correct, incorrect, and omitted trials), and (3) the fixation 
dot period between task trials resulting in 13 regressors in the design matrices. At the single-individual level, a t-contrast at each voxel for each participant was computed to produce statistical images for the contrast TASK- versus BASE-condition for the free recall trial element. At the second level, the resulting contrast images were used to identify the main task effect TASK versus BASE-condition by means of a one sample t-test $(p<.001$, uncorrected). For $\mathrm{PR}$, TASK versus BASE-conditions were contrasted using t-statistics $(p<.001$, uncorrected). Percent signal change values for several regions of interest (ROIs) were extracted applying the software package Marsbar (Version 4.2 [41]). ROIs were extracted according to activation clusters resulting from the contrast "PR vs. reference group" related to "TASK vs. BASE"-condition contrasts. This was done to comprehensibly illustrate core aspects of the present data.

In order to inferentially compare brain activation patterns between PR and the reference group, we decided to follow the methods suggested by Crawford and colleagues [35, 42, 43]. The respective algorithms were implemented as an SPM compliant function that reads the specified individual contrast images and their respective design matrices (SPM.mat files). The appropriate beta images are then loaded as scores for the respective tasks and the calculus is performed. The resulting images were written to disk as spmT image files for use in the result function of SPM $(p<.001$, uncorrected) (see Fehr et al. [10] for further methodological details).

\section{Additional file}

Additional file 1. Neural correlates of free recall of "famous events" in a "hypermnestic" individual as compared to an age- and educationmatched reference group - supplementary information.

\section{Abbreviations}

FMRI: functional magnetic resonance imaging; HS: hyperthymestic syndrome; LTM: long term memory; PR: protagonist; SPM: statistical parametric mapping; S-W-T: Shapiro-Wilk-test.

\section{Authors' contributions}

TF contributed to the experimental design, data acquisition, data analyses and manuscript writing. AS contributed to the neuropsychological testing of PR and manuscript writing. HJM contributed to the experimental design and manuscript writing. PE contributed to fMRI-data acquisition and manuscript writing. MH contributed to the experimental design and manuscript writing. All authors read and approved the final manuscript.

\section{Author details \\ ${ }^{1}$ Center for Cognitive Sciences, University of Bremen, Bremen, Germany. ${ }^{2}$ University of Bremen, Hochschulring 18, 28359 Bremen, Germany. ${ }^{3}$ Center for Advanced Imaging, Universities of Bremen and Magdeburg, Bremen, Ger- many. ${ }^{4}$ Physiological Psychology, University of Bielefeld, Bielefeld, Germany. ${ }^{5}$ AG in vivo MR, University of Bremen, Bremen, Germany. ${ }^{6}$ Hanse Institute for Advanced Study (HWK), Delmenhorst, Germany.}

\section{Acknowledgements}

Not applicable.

\section{Competing interests}

The authors declare that they have no competing interests.

\section{Availability of data and materials}

Please contact the corresponding author to get local offline-access to the original data sets.

\section{Consent for publication}

Not applicable.

\section{Ethics approval and consent to participate}

The study protocol was designed and performed according to the Helsinki Declaration (1964) and was approved by the Ethics Committee of Bremen University. All participants were informed about the procedure, and gave written consent to participate in the experiment.

\section{Funding}

Not applicable.

\section{Publisher's Note}

Springer Nature remains neutral with regard to jurisdictional claims in published maps and institutional affiliations.

Received: 30 May 2017 Accepted: 1 June 2018

Published online: 19 June 2018

\section{References}

1. Baddeley A. Working memory: theories, models, and controversies. Annu Rev Psychol. 2012;63:1-29.

2. Tulving E. Episodic memory: from mind to brain. Annu Rev Psychol. 2002;53:1-25.

3. Tulving E. Episodic memory and autonoesis: uniquely human? In: Terrace HS, Metcalfe J, editors. The missing link in cognition: Self-knowing consciousness in man and animals. New York: Oxford University Press; 2005. p. 3-56.

4. Markowitsch HJ. Psychogenic amnesia. Neuroimage. 2003;20:132-8.

5. Markowitsch HJ, Staniloiu A. Amnestic disorders. Lancet. 2012;380:142940. https://doi.org/10.1016/s0140-6736(11)61304-4.

6. Staniloiu A, Markowitsch HJ. Dissociative amnesia. Lancet Psychiatry. 2014;1:226-41.

7. Parker ES, Cahill L, McGaugh JL. A case of unusual autobiographical remembering. Neurocase. 2006;12:35-49.

8. LePort AKR, Mattfeld AT, Dickinson-Anson H, Fallon JH, Craig EL, Stark CEL, Kruggel F, Cahill L, McGaugh JL. Behavioral and neuroanatomical investigation of Highly Superior Autobiographical Memory (HSAM). Neurobiol Learn Mem. 2012;98:78-92.

9. Amidzic O, Riehle HJ, Fehr T, Elbert T. Focal gamma band activity: the signature of chunks in the expert memory of chess players. Nature. 2001:412:603

10. Fehr T, Weber J, Willmes K, Herrmann M. Neural correlates in exceptional mental arithmetic-About the neural architecture of prodigious skills. Neuropsychologia. 2010;48:1407-16.

11. Fehr T, Wallace $G$, Erhard P, Herrmann M. The functional neuroanatomy of expert calendar calculation: a matter of strategy? Neurocase. 2011:17:360-71.

12. Erdelyi $\mathrm{MH}$, Becker J. Hypermnesia for pictures: incremental memory for pictures but not for words in multiple recall trials. Cogn Psychol. 1974;6:159-71.

13. Bluck S, Levine $L$, Laulhere TM. Autobiographical remembering and hypermnesia: a comparison of older and younger adults. Psychol Aging 1999;14:671-82.

14. Bor D, Duncan J, Wisemann RJ, Owen AM. Encoding strategies dissociate prefrontal activity from working memory demand. Neuron. 2003;37:361-7.

15. Kuo MCC, Liu KPY, Chan CCH. Factors involved in memory encoding and their implications for the memory performance of older adults and people with mild cognitive impairment. World J Neurosci. 2012;2:103-12. 
16. Gobet F, Clarkson G. Chunks in expert memory: evidence for the magical number four ... or is it two? Memory. 2004;12:732-47.

17. Brown NR. Organization of public events in long-term memory. J Exp Psychol Gen. 1990;119:297-314.

18. Scoville WB, Milner B. Loss of recent memory after bilateral hippocampal lesions. J Neurol Neurosur Psychiatry. 1957;20:11-21.

19. Squire LR. The legacy of patient H.M. for neuroscience. Neuron. 2009;61:6-9.

20. Squire $L R$, Wixted JT. The cognitive neuroscience of human memory since H.M. Annu Rev Neurosci. 2011;34:259-88.

21. Wang S-H, Morris GM. Hippocampal-neocortical interactions in memory formation, consolidation, and reconsolidation. Annu Rev Psychol. 2010;61:49-79.

22. Rugg MD, Vilberg KL, Mattson JT, Yu SS, Johnson JD, Suzuki M. Item memory, context memory and the hippocampus; fMRl evidence. Neuropsychologia. 2012;50:3070-9.

23. Rugg MD, Vilberg K. Brain networks underlying episodic memory retrieval. Curr Opin Neurobiol. 2013;23:255-60.

24. Fuster JM. The cognit: a network model of cortical representation. Int J Psychophysiol. 2006;60:125-32.

25. Fuster JM. Cortex and memory: emergence of a new paradigm. J Cogn Neurosci. 2009;21:2047-72.

26. Basar E. The theory of the whole-brain-work. Int J Psychophysiol. 2006;60:133-8.

27. Henke K. A model for memory systems based on processing modes rather than consciousness. Nat Rev Neurosci. 2010;11:523-32.

28. Shohamy D, Turk-Browne NB. Mechanisms for widespread hippocampal involvement in cognition. J Exp Psychol Gen. 2013;142:1159-70.

29. Squire LR. Memory systems of the brain: a brief history and current perspective. Neurobiol Learn Mem. 2004;82:171-7.

30. Nauta WJH. Expanding border of the limbic system concept. In: Rasmussen T, Marino R, editors. Functional neurosurgery. New York: Raven Press; 1979. p. 7-23.

31. Nieuwenhuys R. The greater limbic system, the emotional motor system and the brain. Prog Brain Res. 1996;107:551-80.

32. Shah NJ, Marshall JC, Zafiris O, Schwab A, Zilles K, Markowitsch HJ, Fink $G R$. The neural correlates of person familiarity. A functional magnetic resonance imaging study with clinical implications. Brain. 2001:124:804-15.

33. Tulving E, Markowitsch HJ, Craik FIM, Habib R, Houle S. Novelty and familiarity activations in PET studies of memory encoding and retrieval. Cereb Cortex. 1996;6:71-9.

34. Herholz K, Kessler J, Ehlen P, Lenz O, Kalbe E, Markowitsch HJ. The role of prefrontal cortex, precuneus, and cerebellum during face-name association learning. Neuropsychologia. 2001;39:643-50.

35. Crawford JR, Howell DC. Comparing an individual's test score against norms derived from small samples. Clin Neuropsychol. 1998:12:482-6.

36. Crawford JR, Garthwaite PH. Investigation of the single case in neuropsychology: confidence limits on the abnormality of test scores and test score differences. Neuropsychologia. 2002;40:1196-208.

37. Oldfield R. The assessment and analysis of handedness. The Edinburgh Inventory. Neuropsychologia. 1971;9:97-113.

38. Lehrl S. Mehrfachwahl-Wortschatz-Intelligenztest (MWT-B) [Multiple choice lexis intelligence test]. Balingen: Spitta Verlag; 2005.

39. Friston KJ. Experimental design and statistical issues. In: Mazziotta JC, Toga AW, editors. Brain mapping: the disorders. San Diego: Academic Press; 2000. p. 33-58.

40. Holmes AP, Friston KJ. Generalisability, random effects, and population inference. Neuroimage. 1998;7:754.

41. Brett $M$, Anton J-L, Valabregue R, Poline J-B. Region of interest analysis using an SPM toolbox [abstract] Presented at the 8th International Conference on Functional Mapping of the Human Brain, June 2-6, 2002, Sendai, Japan. Available on CD-ROM in Neuroimage, 16, No 2; 2002.

42. Crawford JR, Garthwaite PH. Testing for suspected impairments and dissociations in single-case studies in neuropsychology: evaluation of alternatives using Monte Carlo simulations and revised tests for dissociations. Neuropsychology. 2005:19:318-31.

43. Crawford JR, Garthwaite PH. Evaluation of criteria for classical dissociations in single-case studies by Monte Carlo simulation. Neuropsychology. 2005;19:664-78.
44. Tulving E, Kapur S, Markowitsch HJ, Craik G, Habib R, Houle S. Neuroanatomical correlates of retrieval in episodic memory: auditory sentence recognition. Proc Natl Acad Sci USA. 1994;91:2012-5.

45. Lepage M, Ghaffar O, Nyberg L, Tulving E. Prefrontal cortex and episodic memory retrieval mode. Proc Natl Acad Sci USA. 2000;97:506-11.

46. Tulving E. Organization of memory: quo vadis? In: Gazzaniga MS, editor. The cognitive neurosciences. Cambridge: MIT Press; 1995. p. 839-47.

47. Dew ITZ, Cabeza R. The porous boundaries between explicit and implicit memory: behavioral and neural evidence. Ann NY Acad Sci. 2011;1224:174-90.

48. Treffert DA. The savant syndrome: an extraordinary condition. A synopsis: past, present, future. Philos Trans R Soc B. 2009;364:1351-7.

49. Marks LE. On colored-hearing synesthesia: cross-modal translations of sensory dimensions. Psychol Bull. 1975;82:303-31.

50. Marks LE, Mulvenna CM. Synesthesia, at and near its borders. Front Psychol. 2013. https://doi.org/10.3389/fpsyg.2013.00651.

51. Rouw R, Scholte HS, Colizoli O. Brain areas involved in synaesthesia: a review. J Neuropsychol. 2011;5:214-42.

52. Svoboda E, McKinnon M, Levine B. The functional neuroanatomy of autobiographical memory: a meta-analysis. Neuropsychologia. 2006;44:2189-208.

53. Piefke $M$, Weiss PH, Markowitsch HJ, Fink GR. Gender differences in the functional neuroanatomy of emotional episodic autobiographical memory. Hum Brain Mapp. 2005;24:313-24.

54. Fink GR, Markowitsch HJ, Reinkemeier M, Bruckbauer T, Kessler J, Heiss W-D. Cerebral representation of one's own past: neural networks involved in autobiographical memory. J Neurosci. 1996;16:4275-82.

55. Insausti R, Annese J, Amaral DG, Squire LR. Human amnesia and the medial temporal lobe illuminated by neuropsychological and neurohistological findings for patient E.P. Proc Natl Acad Sci USA. 2013;110:E195362. https://doi.org/10.1073/pnas.1306244110.

56. Maguire EA, Mummery CJ, Büchel C. Patterns of hippocampal-cortical interaction dissociate temporal lobe memory subsystems. Hippocampus. 2000;10:475-82.

57. Karnath HO. New insights into the functions of the superior temporal cortex. Nat Rev Neurosci. 2001;2:568-76. https://doi.org/10.1038/35086 057.

58. Manning L, Denkova E, Unterberger L. Autobiographical significance in past and future public semantic memory: a case-study. Cortex. 2013:49:2007-20. https://doi.org/10.1016/j.cortex.2012.11.007.

59. De Renzi E, Liotti M, Nichelli P. Semantic amnesia with preservation of autobiographic memory, A case report. Cortex. 1987;23:575-97.

60. Yasuda K, Watanabe O, Ono Y. Dissociation between semantic and autobiographic memory: a case report. Cortex. 1997;33:623-38.

61. Maguire EA, Valentie ER, Wilding JM, Kapur N. Routes to remembering: the brains behind superior memory. Nat Neurosci. 2003;6:90-5.

62. Poldrack RA. Can cognitive processes be inferred from neuroimaging data? Trends Cogn Sci. 2006;10:59-63.

63. Houdé O, Zago L, Mellet E, Moutier S, Pineau A, Mazoyer B, TzourioMazoyer N. Shifting from the perceptual brain to the logical brain: the neural impact of cognitive inhibition training. J Cogn Neurosci. 2000;12:721-8

64. Houdé O, Tzourio-Mazoyer N. Neural foundations of logical and mathematical cognition. Nat Rev Neurosci. 2003;4:507-14.

65. Fehr T. A hybrid model for the neural representation of complex mental processing in the human brain. Cogn Neurodyn. 2013;7:89-103.

66. Addis DR, Knapp K, Roberts RP, Schacter DL. Routes to the past: neural substrates of direct and generative autobiographical memory retrieval. Neuroimage. 2012;59:2908-22. https://doi.org/10.1016/j.neuroimage .2011.09.066.

67. Raz A, Packard MG, Alexander GM, Buhle JT, Zhu H, Yu S, Peterson BS. A slice of $\pi$ : an exploratory neuroimaging study of digit encoding and retrieval in a superior memorist. Neurocase. 2009;15:361-72.

68. Yin L-J, Lu Y-T, Fan M-X, Wang Z-X, Hu Y. Neural evidence for the use of digit-image mnemonic in a superior memorist: an fMRI study. Front Human Neurosci. 2015. https://doi.org/10.3389/fnhum.2015.00109.

69. Patihis L, Frenda SJ, LePort AKR, Petersen N, Nichols RM, Stark CEL, McGaugh JL, Loftus EF. False memories in highly autobiographical memory individuals. Proc Natl Acad Sci USA. 2013;110:20947-52. 